\title{
Martensitic Transformations and Magnetic Properties of Ni-Mn-Sn Heusler Alloy Films
}

\author{
J. Dubowik, A. Szlaferek \\ Institute of Molecular Physics, Polish Academy of Sciences \\ Smoluchowskiego 17, 60-179 Poznań, Poland
}

AND I. GoŚCIAŃSKA

Department of Physics, A. Mickiewicz University

Umultowska 85, 61-614 Poznań, Poland

\begin{abstract}
We report on structural and magnetic properties of $\mathrm{Ni}_{50} \mathrm{Mn}_{50-x} \mathrm{Sn}_{x}$ $(x=12 \div 14)$ films and compare them with those of the bulk $\mathrm{Ni}-\mathrm{Mn}-\mathrm{Sn}$ alloys. Magnetic measurements reveal clear presence of martensitic transformation in bulk alloys but in the flash-evaporated $\mathrm{Ni}-\mathrm{Mn}-\mathrm{Sn}$ thin films martensitic transformation is usually less visible. The best film samples annealed for $1 \mathrm{~h}$ at about $900 \mathrm{~K}$ exhibit clear signs of martensitic transformation: i.e., a small defect in magnetization and a substantial increase in ferromagnetic resonance line width.
\end{abstract}

PACS numbers: 75.50.Cc, 81.30.Kf, 76.50.+g

\section{Introduction}

$\mathrm{Ni}_{50} \mathrm{Mn}_{50-x} \mathrm{Sn}_{x}(x=12 \div 14)$ Heusler alloys (HA) display interesting properties related to martensitic transformations (MT) [1-5] and arising from a coupling between structure and magnetism. They are all associated with MT, which occur below the Curie temperature $T_{C} \approx 320 \mathrm{~K}$ depending strongly on composition $[3,4]$. Ferromagnetic and lattice instabilities have been found to coexist in a narrow $(x=13 \div 14)$ concentration range [2]. The most characteristic feature is that both austenitic and martensitic states are ferromagnetic but with distinct exchange interactions. The aim of this contribution is a search for MT in Ni-Mn-Sn thin films.

\section{Experimental results and their discussion}

The films were deposited onto glass or mica substrates by a flash evaporation technique in an ultrahigh vacuum $\left(10^{-7} \mathrm{~Pa}\right)$ from powders obtained from 
$\mathrm{Ni}_{50} \mathrm{Mn}_{50-x} \mathrm{Sn}_{x}(12 \leq x \leq 14)$ ingots. The ingots were prepared by an induction melting and then annealed at $1273 \mathrm{~K}$ for $24 \mathrm{~h}$. Our bulk Ni-Mn-Sn HA show $L 2_{1}$ (or $B 2$ ) ordering for $x=14$ at room temperature and modulated martensite structure for $x=13$ and exhibit MT typical of this system [4] but the structure of the Ni-Mn-Sn films has been identified as $L 1_{0}$ or a highly (111) textured $B 2$ type of structure [6]. Magnetic measurements were carried out using a vibrating sample magnetometer (VSM) under a magnetic field up to $10 \mathrm{kOe}$ and with ferromagnetic resonance (FMR) from 78 to $350 \mathrm{~K}$ using an X-band spectrometer.

Figure 1a shows the magnetization of $\mathrm{Ni}_{50} \mathrm{Mn}_{50-x} \mathrm{Sn}_{x}$ bulk alloys as a function of temperature in a magnetic field of 100 Oe on cooling (and on cooling and heating for $x=14$ ) in the temperature range between 180 and 350 K. For clarity, we use the arbitrary units in magnetization axis. Ferromagnetic behavior for the sample with $x=14$ is observed in austenitic state below $T_{\mathrm{C}}^{\mathrm{A}} \approx 320 \mathrm{~K}$ but further decrease in the temperature causes a sudden decrease in the magnetization below MT. A hysteretic behavior typical of MT in similar alloys [3] is clearly seen at $260 \mathrm{~K}$. Magnetic behavior for the alloy with $x=13.5$ exhibits two magnetic transitions at $\approx 320 \mathrm{~K}$ and $\approx 250 \mathrm{~K}$, respectively. The high temperature anomaly may result from MT at $310 \mathrm{~K}$, i.e., slightly below $T_{\mathrm{C}}^{\mathrm{A}}$ of $320 \mathrm{~K}$ [2]. The low temperature anomaly has been attributed to a low Curie temperature, $T_{\mathrm{C}}^{\mathrm{M}} \approx 250 \mathrm{~K}$, of martensitic state but its actual origin is not clear [1]. For the alloy with $x=12.5$, the ferromagnetic ordering appears at $\approx 220 \mathrm{~K}$ and is typical of $\mathrm{Ni}-\mathrm{Mn}-\mathrm{Sn}$ alloys in martensitic state with MT much higher than room temperature [1]. Figure 1b shows the magnetization of $\mathrm{Ni}_{50} \mathrm{Mn}_{50-x} \mathrm{Sn}_{x}$ thin films as a function of temperature

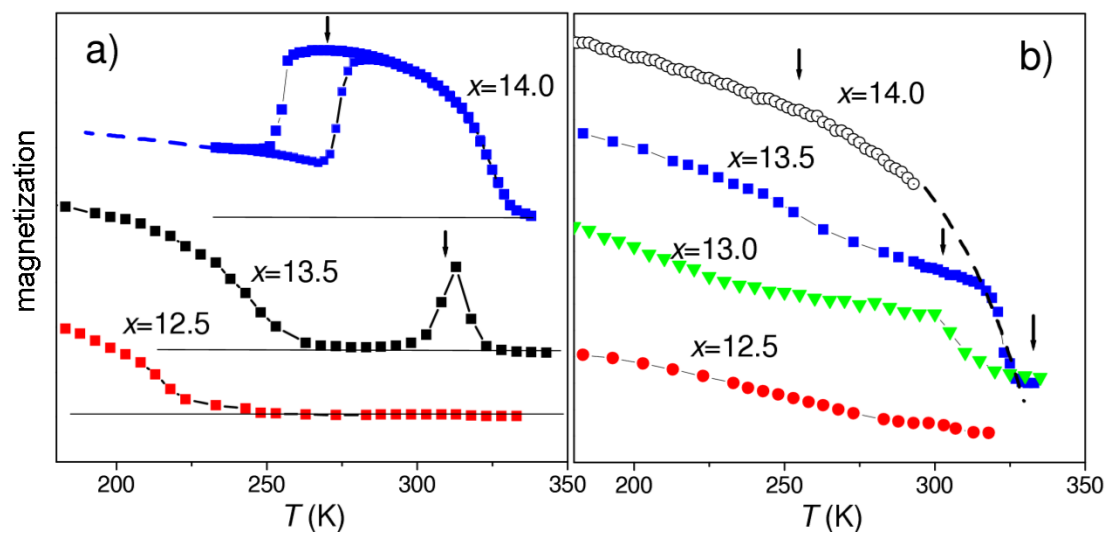

Fig. 1. (a) Temperature dependence of magnetization for $\mathrm{Ni}_{50} \mathrm{Mn}_{50-x} \mathrm{Sn}_{x}$ bulk alloys $(x=12.5,13.5,14)$ under magnetic field of 100 Oe. (b) Temperature dependence of magnetization for $\mathrm{Ni}_{50} \mathrm{Mn}_{50-x} \mathrm{Sn}_{x}$ films $(x=12.5,13.0,13.5,14)$ under magnetic field of 100 Oe. The arrows show MT for bulk alloys and the MT temperature for the alloy with $x=12.5$ is $\approx 370 \mathrm{~K}$. 
in a magnetic field of 100 Oe on cooling in the temperature range between 180 and $350 \mathrm{~K}$. Comparing these data with the data for the bulk samples (Fig. 1a), one can see that the influence of MT on the magnetization behavior is largely smeared and for the film with $x=14$ there is no sign of the characteristic deflection in the magnetization observed in bulk alloys [3]. The reason for such a behavior in the low-field magnetization characteristics of thin $\mathrm{Ni}-\mathrm{Mn}-\mathrm{Sn}$ films is not clear. It may result from a compositional distribution in the thin films or from some constraints of the substrates. As can be seen in Fig. 1a, the MT temperature (shown by arrows) significantly decreases with $x$. However, since the films were annealed at $\sim 900 \mathrm{~K}$ for $1 \mathrm{~h}$ for homogenization and structural ordering, they are believed to be homogeneous. On the other hand, the lack of clear signs of MT in the films may result from their clamping from the substrates since the films are usually constrained by the substrates.
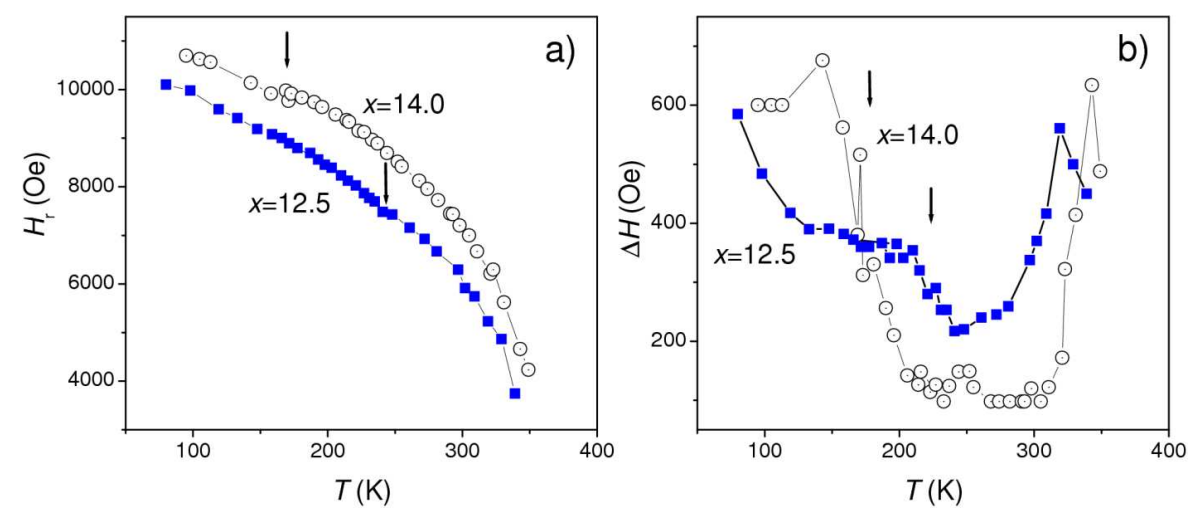

Fig. 2. Temperature dependence of the resonance field $H_{\mathrm{r}}$ (a) and line width $\Delta H$ (b) for $\mathrm{Ni}_{50} \mathrm{Mn}_{50-x} \mathrm{Sn}_{x}$ films ( $x=12.5$ and 14). The measurements were done with the external field applied perpendicular to the film plane.

FMR has been shown useful for tracing structural transformations in thin HA films [7]. We extensively used FMR to reveal MT in our Ni-Mn-Sn films. Recently, we showed that for most of the films the presence of MT is hardly observed and the films exhibit the magnetic properties typical of soft ferromagnets with the FMR line width of 50-200 Oe and the magnetization of $\approx 650$ Gs (extrapolated to $0 \mathrm{~K}$ ) with no clear signs of MT [6]. Figure 2 shows the temperature dependences of the resonance field $H_{\mathrm{r}}$ (a) and FMR line width $\Delta H$ (b) measured for the best of our Ni-Mn-Sn films annealed at $900 \mathrm{~K}$ for $1 \mathrm{~h}$. The measurements were carried out with the magnetic field applied perpendicular to the film plane. For the sake of clarity, we show only the magnetic characteristics of the films with composition $x=12.5$ and 14, respectively. According to the standard FMR relation for the perpendicular configuration $-4 \pi M_{\text {eff }}=H_{\mathrm{r}}-\omega / \gamma$, where $\omega=2 \pi f$ is the angular microwave frequency and $\gamma$ is the gyromagnetic ratio - the temperature 
dependence of $H_{\mathrm{r}}$ (Fig. 2a) corresponds to the temperature dependence of the magnetization. The extrapolated to $T=0 \mathrm{~K}$ value of $4 \pi M_{\mathrm{eff}} \approx 12-3.2 \approx 8.8 \mathrm{kGs}$ $\left(M_{\text {eff }} \approx 700 \mathrm{Gs}\right)$. This value is in a good agreement with the theoretically predicted magnetization with the magnetic moment of $4 \mu_{\mathrm{B}}$ per Mn atom [8] and is the highest among similar HA films exhibiting shape memory effect [7]. In the course of $H_{\mathrm{r}}$ vs. $T$ there are small anomalies indicated by arrows in Fig. 2 (i.e., defects in $\left.4 \pi M_{\text {eff }}\right)$ at $180 \mathrm{~K}(x=14)$ and $250 \mathrm{~K}(x=12.5)$, respectively, and are significantly shifted with respect to the bulk values. It is characteristic that these small defects in $M_{\text {eff }}$ correspond to a very large increase in $\Delta H$ at the same temperatures. We relate such an increase in $\Delta H$ with the existence of MT in our films.

\section{Summary}

Thin $\mathrm{Ni}_{50} \mathrm{Mn}_{50-x} \mathrm{Sn}_{x}(x=12 \div 14)$ films were investigated and compared with the corresponding bulk alloys. Using temperature dependent magnetization and ferromagnetic resonance measurements we found the existence of MT in these films.

\section{References}

[1] Y. Sutou, Y. Imano, N. Koeda, T. Omori, R. Kainuma, K. Ishida, K. Oikawa, Appl. Phys. Lett. 85, 4358 (2004).

[2] T. Krenke, M. Acet, E. F. Wassermann, X. Moya, L. Maňosa, A. Planes, Phys. Rev. B 72, 014412 (2005).

[3] X. Moya, L. Maňosa, A. Planes, T. Krenke, M. Acet, E. F. Wassermann, Mat. Sci Engn. A 438-440, 911 (2006).

[4] K. Koyama, H. Okada, K. Watanabe, T. Kanomata, R. Kainuma, W. Ito, K. Oikawa, K. Ishida, Appl. Phys. Lett. 89, 182510 (2006).

[5] K. Koyama, K. Watanabe, T. Kanomata, R. Kainuma, K. Oikawa, K. Ishida, Appl. Phys. Lett. 88, 132505 (2006).

[6] J. Dubowik, I. Gościańska, Y.V. Kudryavtsev, A. Szlaferek, J. Magn. Magn. Mater. 310, 2773 (2007).

[7] J. Dubowik, Y.V. Kudryavtsev, Y. P. Lee, J. Appl. Phys 95, 2912 (2004).

[8] T. Krenke, M. Acet, E. F. Wassermann, X. Moya, L. Maňosa, A. Planes, Phys. Rev. B 73, 174413 (2006). 